\title{
The training characteristics of severely head-injured patients: a preliminary study
}

\author{
EDGAR MILLER
}

\author{
From the Department of Clinical Psychology, Addenbrooke's Hospital, Cambridge
}

SUMMARY It is argued that an important aspect of rehabilitation after severe head injury lies in training or retraining victims in skills required for independent living. Despite this, very little is known about the training characteristics of head injured patients. It is shown that in the acquisition of a psychomotor skill severely head injured subjects have very poor starting levels as compared to normal controls. They show impressive learning to the extent that they considerably close the gap between themselves and the controls. They also show appreciable transfer of the learned skill from one version of the task to another. The findings are discussed in terms of their implications for further understanding of the training process in head injured subjects in ways that could reflect upon clinical practice in rehabilitation.

There is no doubt that severe head injury can have profound long-term effects on the victim. Good follow-up data is sparse but the available evidence suggests that an appreciable proportion fail to attain a level of social and occupational adjustment commensurate with their status prior to the injury. ${ }^{1}$ It has been found by Bond ${ }^{2}$ amongst others that the psychological consequences of head injury (changes in memory, personality, etc) typically have a more deleterious effect on later adjustment than the physical impairments (hemiplegia, epilepsy, etc). Prominent among the psychological effects of severe head injury are impairments in memory or learning or both. The experimental work on these deficits, which has been heavily biased towards studies of verbal learning and memory, has recently been reviewed by Schacter and Crovitz. $^{3}$

There is little that can be done by conventional medical therapies to restore lost functional capacity after brain damage, although there are means of ameliorating some of the physical consequences such as epilepsy. In general terms much of the onus in rehabilitation must be thrown upon ways of training or retraining the victim in skills necessary for everyday living and re-entry to independent life in the community. Despite the considerable

Address for reprint requests: Dr E Miller, Department of Clinical Psychology, Addenbrooke's Hospital, Cambridge CB2 2QQ.

Accepted 13 March 1980 amount of work on verbal learning and memory after head injury ${ }^{3}$ there has been little or no interest in the training of non-verbal skills. Studies of training might prove to be of much more direct relevance to rehabilitation.

The present paper reports some early data from what is intended will be a much more extensive investigation of the training characteristics of severely head injured patients. The writer knows of no directly relevant previous work on head injury. Because of this it was decided to start with the general hypothesis that severely head injured subjects would show similarities with another severely "brain damaged" population, the severely mentally subnormal, with regard to which there is a substantial literature on training.

Briefly it appears to be the case that the severely mentally subnormal have very poor starting levels on most tasks. Given extensive and, if necessary, appropriately designed training opportunities they do learn. On some surprisingly complex tasks they can eventually achieve approximately the same level of performance as normal subjects. What is also encouraging is that when matched for the initial level of learning the severely subnormal retain skills that they have learned as well as normal controls. An extensive discussion of these issues can be found in Clarke and Clarke. ${ }^{4}$ Whilst no strong hypothesis is being advanced that the training characteristics of the severely brain injured and the severely subnormal will be similar 
in every way it is not unreasonable to assume that the work with the mentally subnormal will provide a useful model and indication of what might be expected in looking at the problem of training the severely head injured patient.

In line with this general argument the present experiment examined the learning and transfer of training of head injured subjects using a moderately difficult psychomotor task that had been used in previous work with the severely subnormal.

\section{Subjects and methods}

The experimental group consisted of eight male patients who had suffered severe head injuries (approximate PTAs ranged from two to eight weeks). All were young adults (aged 17 to 35 years with a mean age of 23.3 years). They were tested several months (range approximately three to 14 months) after their injury and when they were clearly out of the period of PTA. All were inpatients in the Rehabilitation Unit of Addenbrooke's Hospital. Control subjects were neurologically normal and were matched closely for age with the experimental group. On average the control group was better educated and of higher socio-economic status but there is no reason to suppose that any small biases which these differences might have introduced would be of such an order as to alter the general picture given by the results.

The experimental task was based on the Minnesota Spatial Relations Test which was here used as a learning task rather than as a "test" as originally intended. The equipment consists of four separate rectangular boards (about 79 $\times 22 \mathrm{~cm}$ ) each containing 58 differently shaped holes. The subject's task in each case was to fit 58 shapes into their corresponding holes as quickly as possible. The boards are all of similar, but by no means exactly equal, difficulty and were therefore administered in a balanced order to control for the small differences in difficulty.

Subjects had one trial per working day (Monday to Friday). They had five trials on the first board (Board A), followed by five trials with the second board, and so on (Boards $B$ to D) until all four boards had been completed ( 20 trials in ali). The measure used was the time in seconds required to fit all the pieces into the board. All subjects carried out the task seated at a table.

\section{Results}

These are shown in graphical form in fig 1 .

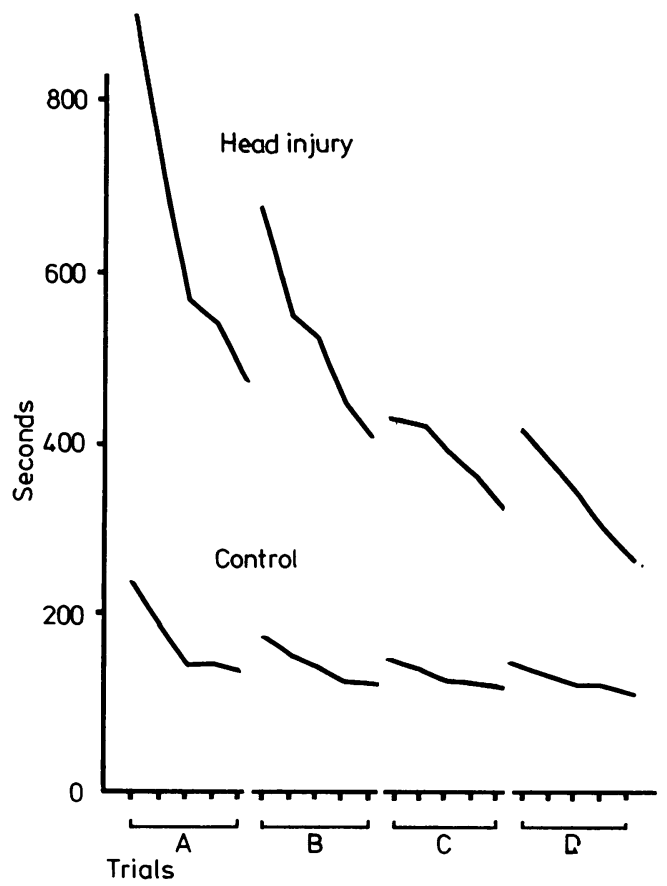

Fig 1 Mean times on successive trials on the four boards of the Minnesota Spatial Relations Test.

Because of the very large effects observed statistical analysis is somewhat superfluous but it ought perhaps to be confirmed that the main effects described were all highly significant when subjected to analysis of variance.

It can be seen that the two groups start at very different levels with both showing improvements with practice and good transfer of training from one board to the next. At the end of the experiment the controls appear to be very close to an asymptote whereas the head injured group are still improving and have considerably closed the gap between themselves and the controls.

As shown in fig 1 it looks as if the head injured group is paradoxically learning faster than the control group. This is almost certainly due to the fact that the control group, having started at a level much closer to their optimal level of performance, have less room for improvement. In order to examine relative speeds of learning it is necessary to match the two groups for initial starting level. It proved possible to match fairly closely the mean time taken by four of the head injured subjects on the first trial of their fourth board with that for four control subjects on their first board. Fig 2 shows 


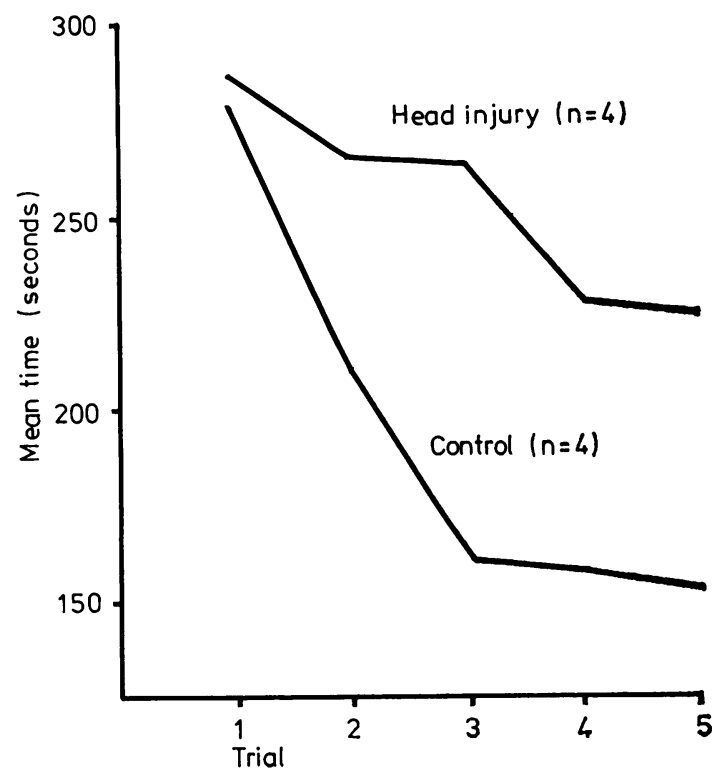

Fig 2 Relative rates of learning in two subgroups when matched for initial performance levels.

the performance of these two subgroups on the fourth and first boards respectively. The apparent relative rate of learning is now reversed with the head injured group being slower to learn.

\section{Discussion}

At face value the results of this experiment suggest certain conclusions and prompt a number of questions. The results also have a simple, but possibly useful, practical implication for rehabilitation.

The conclusions are as follows. Despite having extremely poor starting levels as compared with controls, the severely head injured group shows appreciable learning with continued practice. There is impressive transfer of training from one board to the next which is encouraging since most learned skills have to be applied in situations which are not identical with those under which the skill was learned. Over the time of the experiment the performance of the head injured group has got much closer to that of the controls and at the end the head injured group is still showing appreciable improvement whilst the controls appear to have reached their asymptote. This raises the possibility that given more extensive training the head injured group might get very close to the performance level of the controls. It is likely that the head injured subjects would never reach the same speed as the controls and their slower reaction times would be one factor likely to preclude this. ${ }^{5}$ Nevertheless considerable further closing of the gap might be expected.

When both groups are matched for initial level, as in fig 2, the indication is that the head injured group improve more slowly and therefore may be showing the effects of a learning impairment. This is not an unlikely state of affairs but the problem in interpreting fig 2 is that in controlling for starting levels other things have ceased to be matched. The control group is seen over its first few trials and the other group over its last. Work on the training of normal subjects has indicated that even though the overall nature of the task may not change throughout the training period, the actual components of the skill that are critical in performing the task do vary with the stage of training. ${ }^{6} 7$ Thus it is possible that the comparison depicted in fig 2 is contaminated because the two groups were not actually learning the same components of the overall task.

It can be argued that at least part of the improvement in the head injured group could have been due to spontaneous recovery since many of this group were not tested after the time at which spontaneous recovery could be presumed to have been completed. Although this possibility cannot be entirely discounted, there are reasons for supposing that spontaneous recovery could not have had a large enough effect to invalidate the general conclusions. Firstly, inspection of the data shows no relationship between the time elapsed from the accident to starting the experiment on the one hand, and the apparent rate of learning on the other. Secondly, general clinical observation testifies that the general rate of improvement shown by the head injured group on the experimental task is vastly in excess of their rate of change in any other area of functioning that might be considered to be affected by spontaneous recovery.

In general the results are very comparable with those obtained from subjects with severe mental subnormality. ${ }^{4}$ They vindicate the use of work on training in subnormality as at least a starting point for examining the problem of training in severe head injury. Workers attempting to habilitate the mentally subnormal have been put off in the past by the patient's extremely poor starting level on any skill. The 
natural assumption is that if the starting level is very bad then little improvement can be expected. The demonstration that extensive and appropriately designed training programmes could achieve good results despite very poor initial levels gave the confidence to teach a much more extensive range of skills to this patient population. The same situation could be true in the rehabilitation of the severely head injured.

The results of this experiment pose further questions. That of the ultimate possible level after even more prolonged training has already been mentioned. If severely head injured subjects are slow to learn as suggested by fig 2 it may be that their rate of learning can be enhanced by suitable training strategies. Breaking the overall task down into separate steps has proved effective in training the severely subnormal and might similarly be useful with the head injured. A final and important question relates to the long-term retention of learned skills. There is little point in training patients inskills that are rapidly forgotten and the headinjured subject's ability to remember what he has learned needs to be examined.

The apparatus used in this study was loaned by Professor ADB Clarke of the University of Hull and his assistance is gratefully acknowledged. Thanks are also due to the physicians responsible for the Rehabilitation Unit at Addenbrooke's Hospital for permission to study patients under their care.

\section{References}

1 Miller E. The long-term consequences of head injury: a discussion of the evidence with special reference to the preparation of legal reports. $\mathrm{Br}$ $J$ soc clin Psychol 1979; 18:87-98.

2 Bond MR. Assessment of the psychosocial outcome after severe head injury. In: Ciba Foundation Symposium No 34: Outcome of Severe Damage to the Central Nervous System. Amsterdam: Elsevier, 1975: 141-57.

3 Schacter DL, Crovitz HF. Memory function after closed head injury: a review of the quantitative research. Cortex 1977; 13:150-76.

4 Clarke AM, Clarke ADB. Mental Deficiency: the Changing Outlook. Third edition. London: Methuen, 1974.

5 Miller E. Simple and choice reaction time following severe head injury. Cortex 1970; 6:121-7.

6 Fleishman EA. Individual differences and motor learning. In: Gagne RM, ed. Learning and Individual Differences. Columbus: Merrill Books, 1968: 143-61.

7 Fleishman EA, Fruchter B. Factors structure and predictability of successive stages of learning morse code. J Appl Psychol 1960; 44:97-101. 